\title{
ARTICULO
}

Revista Derecho - Año 2 edición 3: 195 - 208

Web: http://www.revistaderecho.pe E-mail: editorial@revistaderecho.pe ISSN 2313-6944

\section{EL DERECHO A LA CONSULTA PREVIA EN UN ESTADO NEOLIBERAL}

\section{Choquehuanca Huarcaya Ronald Alan \\ Quispe Chura Alex*}

\section{INFORMACIÓN DEL ARTICULO}

Art. Recibido: 04/02/16

Art. Aceptado: 01/06/16

Art. Publicado: 18/12/18

PALABRAS CLAVE:

Consulta previa Ideología

Neoliberalismo Sistemismo

\section{RESUMEN}

El presente ensayo trata sobre las políticas públicas del estado, de protección a los pueblos indígenas, que se basan solo en el derecho a la consulta formal; sin embargo, este derecho tiene como finalidad llegar a un acuerdo a través de una negociación "libre e informada"; dicha forma de protección, es necesaria pero no suficiente; ya que todo sistema social está influido por muchos factores; factores que en un Estado dirigido por una ideología neoliberal, no son tomados en cuenta, lo que cuenta es la maximización de beneficios del individuo.

\section{THE RIGHT TO PRIOR CONSULTATION IN A NEOLIBERAL STATE}

ARTICLE INFO

Article Received: 04/02/16

Article Accepted: 01/06/16

Article Published: 18/12/18

KEY WORDS:

Prior consultation

Ideology

Neoliberalism

Systemism

\section{ABSTRACT}

The present essay studies the public policy of the state, those on protection to indigenous people, which are based only on the right to formal consultation; however, this right has the purpose to achieve an agreement through a "free and informed" negotiation; this form of protection, is necessary but not sufficient; because every social system is influenced by many factors; factors that in a state run by a neoliberal ideology are not taken into account, what counts is the maximization of individual benefits.

Estudiantes de la Universidad Nacional del Altiplano-Puno, FCJP, Escuela Profesional de Derecho. 


\section{INTRODUCCIÓN}

En el contexto político, por el que nuestro país viene pasando, se puede advertir una intensa influencia del sistema neoliberal. Mas, cuando el instrumento predilecto de dicha ideología, son las normas que se emiten por nuestros legisladores. Tales como: la denominada "Ley Pulpín" (Nuevo régimen de la ley juvenil laboral), "ley SERVIR", asimismo, las modificatorias al código civil, como es el artículo que regula la defensa extrajudicial de la posesión; así como las modificatorias al código procesal civil, y entre otros. Estas normas jurídicas, que vienen formando parte de nuestro ordenamiento jurídico, no son más que utilizadas para legitimar la ideología imperante en nuestro país.

Pues al considerarse al Derecho como una rama no ajena a la política, más al contrario se desarrolla, si y solo si, existe esta última, debe ser analizado tomando en consideración distintos factores como son; político, ambiental, además, económico y sociológico. Es el caso del presente trabajo de investigación, que no se remitirá, a realizar una interpretación normativa de la ley de la consulta previa -se considera al derecho dentro del contexto social-, sino, analizará el problema del derecho a la consulta previa.

Para lo cual, en un principio, determinaremos las condicionantes, entorno a la formación de la decisión, de la consulta previa, así como la influencia del factor económico, alimenticio, educación, seguridad, ambiental y político.
Ha esto se agrega, que los factores antes mencionados, serán analizados en base a determinados casos concretos, que serán desarrollados en su oportunidad.

Asimismo, se realizará una exposición crítica de la justificación ideológica del derecho a la consulta previa, para determinar su ideología política y su influencia en la misma. Esto para circunscribir nuestro problema.

Por último, en razón a los objetivos precedentes, que serán necesarios para un entendimiento sistemático del tema, se buscará determinar la valoración de la decisión que se da en el trámite de la concesión minera; esto, para finalmente dar una respuesta a la pregunta ¿apariencia o realidad?

2. DETERMINANDO LAS
CONDICIONANTES,
ENTORNO A LA FORMACIÓN
DE LA DECISIÓ, DE LA
CONSULTA PREVIA

El objetivo, al abordar la consulta previa, es comprender todo lo que ella en realidad denota. Esto es, ir más allá de lo meramente normativo, para así conocer la realidad a la que hace referencia y en donde encuentra su razón de ser. Y pensamos que ese debería ser el objetivo de cualquier trabajo que no pretende ser reduccionista ni ajeno a la realidad social sistémica (Bunge, 2012).

Y con esto, no pretendemos desmerecer los estudios normativos que se han realizado hasta ahora, sino solo queremos dejar en claro, y criticar al mismo tiempo, las investigaciones 
sectoriales con humos o conclusiones aparentemente integrales. Que llevan solo al desatino o fracaso, cualquier pretendida investigación que busca conocer o explicar un problema inmerso en un sistema.

Es por eso, que no haremos una excepción a la regla. Ya que solo partiendo de ese enfoque se podrá comprender la connotancia: natural (biológica y ambiental), económica, política y cultural que la consulta previa encierra. $\mathrm{Y}$ en donde pensamos está la parte medular que puede ayudarnos a comprender este problema.

Sobre todo, si somos de los que piensan que la consulta previa, no es un problema aislado ni mucho menos uno que deba ser investigado al margen de los demás problemas. Sino, uno que debe ser estudiado sistémicamente, partiendo de la concepción filosófica de que el mundo natural o social está compuesto de supra-sistemas, sistemas y subsistemas (Bunge, 2009). $\mathrm{Y}$ que pueden sintetizarse (en el caso del mundo social) en los siguientes subsistemas: el subsistema natural (biológico y ambiental), económico, político y cultural.

Sin embargo, haciendo un pequeño paréntesis para no dejar al lector en una situación de incomprensión, así como para despejar cualquier duda que hubiese acerca del estudio sistémico - pero sin pretender desarrollarlo por no ser el espacio adecuado, así como para no apartarnos de nuestro problema y de nuestro objetivodiríamos que el enfoque sistémico, de acuerdo con Bunge (2009), parte de la premisa de que la sociedad es un sistema (por ejemplo, cada individuo es parte de una familia, parte de un grupo de estudios, parte de un determinado grupo religioso, parte de la red de amigos, parte de una pandilla, parte de una empresa, parte de una universidad o escuela, parte de un partido político etc.), es decir es una red de vínculos estrechamente relacionados e influenciados -en otras palabras, es imposible comprender las acciones de un individuo sin considerar los sistemas de los cuales forma parte-; por tanto cualquier intento de estudio de un elemento (o individuo) de toda esta red conectada, debe hacerse en ese sentido.

Es por ello, que partiendo de un enfoque sistémico, a continuación hacemos un desarrollo de los factores que según la hipótesis que planteamos, intervienen en la formación de la decisión en relación a la consulta previa. Como son: el factor económico, el factor alimenticio, el factor educación, el factor seguridad, el factor ambiental y el factor político.

\section{La influencia del factor econó- mico, alimenticio, educación, seguridad, ambiental y políti- co, en la consulta previa}

Antes de desarrollar los seis factores que intervienen en la formación de la decisión en la consulta previa, es necesario precisar ¿qué es el derecho a la consulta previa? Para, a partir de esa premisa mayor, disgregar todos los elementos de ella. Y entender el todo a través de sus partes, sus vinculaciones o influencias (que existen entre las 
partes y, las partes y su unidad). Tal como lo exige el método analítico.

Entonces, partiendo de lo afirmado, definimos a la consulta previa como "la facultad de la que gozan los pueblos indígenas y tribales, para que, dentro de un proceso de diálogo intercultural, puedan escuchar al Estado y viceversa, sobre los proyectos que el gobierno tiene pensado aplicar, con el objeto de evaluar, estudiar y analizar los posibles cambios, beneficios y/o perjuicios que dicho proyecto tendría sobre su vida y cultura" (Flores, 2011, pág. 3). Asimismo, esta consulta debe estar dirigida a obtener su consentimiento libre e informado. Tal vez esta no sea la mejor definición, pero es la que mejor resume o sintetiza todo lo referido a la consulta previa.

Para un desarrollo más ordenado de los factores que intervienen en la consulta previa, es necesario también un panorama distribuido en etapas, que ayude a comprender mejor el problema. Por lo que, nos remitimos a las pautas que el Tribunal Constitucional ha brindado en relación con las etapas en que se debe estructurar la consulta previa. Donde se indica que:

En primer lugar, el inicio del proceso debe ser la determinación de la medida legislativa o administrativa que pueda afectar directamente a un pueblo indígena, tarea que debe ser realizada por la entidad que se propone implementar la medida. En segundo lugar, se deben determinar todos los pueblos indígenas que puedan ser afectados, a fin de notificarlos de la medida y de la posible afectación. En tercer lugar, se debe brindar un plazo razonable para que los pueblos indígenas puedan formarse una opinión respecto a la medida por implementar, tras lo cual se pasará a la etapa de negociación propiamente dicha. En cuarto lugar, si el pueblo indígena involucrado se encuentra de acuerdo con la medida consultada, entonces concluye la etapa de negociación. De lo contrario, si es que el pueblo indígena rechaza la medida propuesta, precluye la primera etapa de negociación, con lo cual se pretende hacer visibles los aspectos sobre los cuales existe desacuerdo. En este punto, la medida no podrá ser implementada. Para poder lograrla se tendrá que iniciar una segunda etapa de negociación dentro de un plazo razonable. Si a pesar de los esfuerzos realizados por las partes no se alcanza consenso alguno, solo entonces el Estado podrá implementar la medida, atendiendo en lo posible a las peticiones del pueblo afectado, como se ha recogido en el Fundamento 41 de la STC 0022-2009-PI/TC (Merino \& Lanegra, 2013, pág. 107).

Ahora bien, partiendo de la definición de la consulta previa -sobre todo de la parte que refiere: la consulta debe estar dirigida a obtener su consentimiento libre e informado-, en relación a los puntos tres y cuatro de la secuencia fáctica desarrollada por el 
Tribunal Constitucional -que versan sobre la etapa de consulta y de formación de una opinión respecto a la medida por implementar, y sobre la etapa de consentimiento o acuerdo, respectivamente-. Lo que a continuación sigue es, explicar y justificar, la influencia del primer factor. El factor económico. Para el que, no sería mala idea comenzar con una interrogante.

Si el pueblo tribal (denominación que le da el convenio 169 de la OIT), tuviese todos los recursos necesarios para satisfacer sus necesidades, o si el Estado a través de políticas públicas contribuyera a que estos no tengan dificultades para satisfacer sus propias necesidades -como: trabajo remunerado (que pueda ayudar a satisfacer otras necesidades básicas), educación, salud, seguridad y condiciones para un mejor aprovechamiento, de sus recursos naturales o comerciales-. Aun así, ¿estos darían su consentimiento para que se explote los recursos (como el oro, la plata, el cobre, el petróleo etc.), pese a que sepan que causarán daños ambientales irreversibles? Pensamos que no. Sin embargo, si el lector nos pregunta en que sustentamos esto, el respaldo empírica de la influencia o no de este factor, en la formación de la decisión, la daremos más adelante y en relación con los otros factores.

En cuanto al factor alimenticio, cabe también hacer la pregunta. Si el Estado a través de sus políticas públicas contribuyera en lo posible a que estos pueblos satisfagan su necesidad, o en último caso, satisfaga su necesidad de alimentación. (Que al estar partiendo de un enfoque sistémico, está relacionado con el trabajo y los recursos económicos destinados a cubrir el hambre). Tal vez, estos estos pueblos, evaluarían mejor la situación -en relación a otras opciones- antes de dar su consentimiento. Sin embargo, de seguro darán su consentimiento. Aunque su satisfacción esté condicionada al consentimiento para la explotación de su ambiente Ya que la alimentación es vital para seguir viviendo. Esta sería la razón, por la que las empresas mineras para conseguir el consentimiento de los pueblos proponen comedores, programas de abastecimiento de recursos básicos alimenticios, trabajo remunerado, carreteras o vías de comunicación, etc. Sin embargo, dejemos esto aquí.

Con respecto a la educación, que es también otro factor que interviene en la consulta previa, a través del cual se practican muchas formas de condicionar el consentimiento de los pueblos tribales, como por ejemplo: frente a la desidia del Estado, la empresa -que pretende explotar los recursoscrea escuelas, brinda educación básica, y otorga oportunidades educativas y profesionales, a cambio de que les permitan explotar recursos naturales. Pero, si el Estado contribuye o ayuda a cubrir esta necesidad básica, que además es necesaria para construir una mejor sociedad, tal vez las cosas se reviertan; y llegado el momento estos pueblos opten por cualquiera de las opciones, que se puede traducir en no dar su consentimiento, a favor de la explotación de recursos como: el acuífero, gasífero, minero etc. 
Por otro lado, solo será necesario realizar algunos alcances sobre el factor seguridad, para justificar la influencia de este en el proceso de consulta previa.

Primero, que para su verificación empírica, se deberá partir por entender por seguridad: "el conjunto de condiciones materiales de existencia, tales como alimento, vivienda, empleo, asistencia social, protección de la violencia, un ambiente limpio y pacífico y una pensión para la tercera edad" (Bunge, 2009, pág. 144). Ya que, como refiere Bunge (2009), la seguridad posee tres dimensiones principales: ambiental, biológica y económica.

Segundo, que si el lector siente que solo se está remarcando la influencia del factor alimenticio y económico. Aclaramos que éste no engloba en sí al factor alimenticio y económico, sino solo en la parte que se refiere a la seguridad alimenticia y a la seguridad económica. Ya que una cosa es dar o tener trabajo y otra, muy distinta, es tener seguridad laboral.

Hecha estas precisiones y retomando el rumbo de nuestro propósito, cabe señalar, que las dimensiones en las que se pueden palpar mejor la influencia de este factor -para evitar su confusión con algún otro-; son en su dimensión ambiental, donde hace referencia a la protección contra las catástrofes ambientales, la contaminación industrial; y en su dimensión biológica, que consiste en la protección contra toda clase de violencia. Por lo que no se puede negar, que este factor, por medio de sus dimensiones, suele ser aprovechado por las empresas para condicionar la decisión de los pueblos tribales.

Ahora, pasemos a un factor que es bastante conocido y estudiado, por su influencia evidente en la formación del consentimiento de los pueblos tribales, que es el ambiental. Cuya prueba empírica de su tremenda influencia es: lo sucedido en Arequipa (caso Tía María) y en Puno (Santa Ana). Así como, los otros tantos ejemplos, que justifican su influencia, razón por la que, evitamos justificar este factor.

Por último, tenemos al factor político, que tiene que ver con la organización y debida participación -directa o a través de representantes- de los pueblos afectados, en todo el proceso de consulta previa; por ejemplo, en el conflicto minero en Santa Ana (Puno), de acuerdo con las investigaciones realizadas por Pinto (2013), hubo un punto que llama mucho la atención, además de los puntos vinculados con la superposición de la concesión sobre zona protegida y con la titularidad de una empresa extranjera en zona de frontera, que contraviene el Art. 71 de la Constitución. Pues, entre los pobladores del distrito de Huacullani y Kelluyo, no habría existido una adecuada organización, tanto en la primera consulta como cuando se hizo frente a la empresa Canadiense Bear Creek Mining Company. Muestra de ello, es la división entre ambos pobladores, y los posteriores conflictos que se produjeron.

Sin embargo, toma importancia este hecho $-\mathrm{y}$ sobre todo para el 
investigador-, en el instante en que se verifica que lo sucedido en Bagua $y$ en otros lugares, no fueron ajenos a lo que sucedió en el conflicto minero en Santa Ana. ¿De qué se ha estado hablando? De que en la mesa de dialogó o consulta, si el interlocutor no es quien dice ser -esto es, si no tiene legitimidad y si no se encuentra respaldado por una buena organización social (informada y participativa)-, se deberá correrse el riesgo de que la consulta no se haya hecho con el interlocutor correcto.

Por tanto, si no se obtiene una respuesta libre e informada (en razón a la concurrencia positiva de los seis factores, que son condición necesaria para que sea libre e informada) de nada servirá la consulta. Porque no cumplirá con el propósito, para el que fue creado.

En resumen: partiendo de una perspectiva sistémica. Si positivamente concurrieran los seis factores en el proceso de consulta previa; esto es, que el estado haya realizado políticas públicas, haya emitido normas en paquete (apoyadas en la tecnología social, como esta a su vez, en conocimientos científicos u otros conocimientos), con el propósito de dar condiciones favorables (por no decir equitativas), para el proceso de consulta previa, ¿Estos darían su consentimiento para que se explote los recursos (como el oro, la plata, el cobre, el petróleo etc.), pese a que sepan que causarán daños irreversibles?

Nos atrevemos a afirmar que no. Sin embargo, si fuera un sí, estamos seguros de que por lo menos se explotará, bajo condiciones mucho más óptimas que las actuales. Claro que es una hipótesis; pero que, al menos encuentra respaldo indirecto, en la hipótesis sobre la influencia de seis factores en el proceso de consulta previa, que encuentra respaldo empírico: en lo sucedido en el caso Corani en Carabaya (factores que intervinieron: económico, alimenticio, seguridad, político, educación), en lo sucedido en el caso Santa Ana en Chucuito (factores que intervinieron: político, ambiental, económico); así como, en lo sucedido en el caso Bagua en Amazonas (Damonte \& Ramírez, 2012), Conga en Cajamarca y Proyecto Tía María en Arequipa.

\section{JUSTIFICACIÓN IDEOLÓGICA DEL DERECHO A LA CONSULTA PREVIA}

La justificación ha de entenderse en su significado general que es de proporcionar un fundamento o razón para un constructo o una acción, responde a la pregunta ¿por qué? Debemos realizar algunas precisiones respecto a lo que se entiende por ideología, concebimos a la ideología como un sistema de creencias en particular de juicios de valor y declaraciones de objetivos (Bunge, 1985). Estas creencias están dirigidas a organizar y movilizar personas de cierta clase para trabajar o luchar y poder alcanzar ciertas metas individuales o sociales (Bunge, 1999). Mario Bunge (2009), señala que existen tres tipos de ideologías: global, religiosa y sociopolítica, esta última es una perspecti- 
va o concepción general del mundo social. Vale decir, las proposiciones (principios) generales, juicios de valor y propuestas prácticas de una ideología sociopolítica se refieren al orden social y a los modos de conservarlo o transformarlo. Responden a cuestiones acerca de la sociedad, así como de programas de acción social. Sistema de valores concernientes a la buena sociedad y a la conducta social correcta, al conjunto de medios, casi todos prácticos, para alcanzar programas de acción social. Se debe tener en cuenta que casi todos nos enfrentamos a los acontecimientos políticos con algún preconcepto ideológico.

Respecto a la ideología imperante en nuestro país, asumimos que es la ideología neoliberal; según David Harvey (2007), el neoliberalismo es, ante todo, una teoría de prácticas político-económicas que afirma que la mejor manera de promover el bienestar del ser humano consiste en no restringir el libre desarrollo de las capacidades y de las libertades empresariales del individuo dentro de un marco institucional caracterizado por derechos de propiedad privada fuertes, mercados libres y libertad de comercio; asimismo, se debe saber que la escuela austríaca junto con la escuela de Chicago fundaron o, mejor dicho, otorgaron "corpus teórico" e ideológico a lo que popularmente se conoce como neoliberalismo; sintetizando podemos afirmar que el neoliberalismo es una corriente de pensamiento económico y político que predica (Gallardo, 2014):
1. Liberalización del comercio. Que no se pongan trabas a las importaciones, que no se pongan cupos ni límites a las entradas y salidas de productos, que las tarifas sean uniformes, etc.

2. Liberalización de la inversión extranjera. Que todo extranjero que quiera y pueda, compre sin límites en el país, y viceversa, que todo nacional pueda comprar en el extranjero.

3. Privatización de las empresas públicas. Según el neoliberalismo, el Estado debe reducirse al mínimo, y deshacerse de pagar o costear servicios innecesarios (que suelen ser pagados con los impuestos). Es más, lo mejor que puede hacer el Estado es deshacerse de todos los servicios públicos y dejar que los gestionen de manera más eficaz (o eso dicen), empresas privadas, que harán mucho mejor y más baratos esos servicios que antes hacía el Estado.

4. Desregularización. Abolición de regulaciones (leyes y normas legales) que impidan la entrada en los mercados, o que restrinjan la competencia, y que las instituciones financieras no sean muy severas en su vigilancia.

5. Garantías legales para los derechos de propiedad privada. Que el Estado garantice que lo mío es mío, y lo tuyo es tuyo. De hecho, esta es una de las pocas funciones para las que debería 
quedar el Estado, según el neoliberalismo.

6. La competitividad es buena. La competitividad es la razón por la cual las empresas se esfuerzan por mejorar y dar cada vez más y mejores productos a un mejor precio: es la fuerza motriz tras la cual está el ansia de mejorar más y más del capitalismo. Esa es la gran ventaja de lo privado frente a lo público (lento, ineficaz y sin ganas de mejorar, porque como no tiene nada que vender ni ganar, no pone empeño en ello.

7. Menos Estado. Minarquismo, el gobierno mínimo necesario, para que no cueste mucho en impuestos, y sólo para lo que de verdad haga falta (policía, jueces, ejército, etc.).

8. Y más individualismo. El individuo y sus deseos son lo más importante para el neoliberalismo. La persona individual (y no la "comunidad" o el Estado), es la medida de todas las cosas. Es el llamado "egoísmo sano": mi felicidad va por delante (y, por tanto, mi libertad).

Se debe hacer una aclaración, se ha simplificado mucho las propuestas de esta corriente de pensamiento, y que muchas sub-corrientes no tienen algunas de estas características o tienen otras muy distintas o las enuncian de forma diferente. Se está resumiendo a grandes rasgos para hacer una visión de conjunto. Todas estas teorías comparten los supuestos principales, especialmente los referentes al egoísmo y la maximización de los beneficios, así como la deseabilidad y realidad del mercado libre.

Consideramos que estos son los postulados principales, con los cuales no estamos de acuerdo, y que generalmente colisionan con las ideas de los pueblos indígenas (otras formas de entender el mundo). Asimismo estamos convencidos que se debe seguir una ideología sustentada en la ciencia. La economía llamada popularmente neoliberal muchas veces ha sido catalogada como seudocientífica.

En el tema que nos preocupa y ocupa, habiendo adoptado definiciones, la pregunta que nos formulamos es ¿Por qué consultar? La respuesta más simplista desde el Derecho sería: porque la Ley lo establece; de acuerdo al artículo 6 del Convenio 169 en su inciso establece que "Al aplicar las disposiciones del Convenio, los gobiernos deberán: a) consultar a los pueblos interesados, cada vez que se prevéan medidas legislativas o administrativas susceptibles de afectarles directamente".

Por su parte, en la Ley del derecho a la consulta previa (Ley $\mathrm{N}^{\circ}$ 29785, 2011), (en adelante la Ley) en su artículo 3 (Finalidad de la consulta) menciona que la consulta se realiza "respecto a la medida legislativa o administrativa que les afecten directamente, a través de un diálogo intercultural que garantice su inclusión en los procesos de toma de decisión del Estado (...)". Y algo más, en el artículo 15 se señala que, de no 
alcanzarse un "acuerdo" entre ambas partes "corresponde a las autoridades estatales adoptar todas las medidas que resulten necesarias para garantizar los derechos colectivos de los pueblos indígenas (...)”. En buena cuenta, el Estado tiene la potestad de decidir al final lo que crea conveniente. Para que no quepa dudas el reglamento ( D.S. $\mathrm{N}^{\circ}$ 001-2011-MC, 2011) de la ley de consulta en su artículo 1 inc. 1.5 señala que el resultado del proceso de consulta no es vinculante.

De una interpretación sistemática podemos concluir que no existe un derecho de veto (¿y la Autodeterminación? jel reconocimiento como Naciones? Ni la ley, ni el reglamento lo mencionan), este corto recorrido normativo nos permite conocer la finalidad de la emisión de dichas normas. Estamos convencidos de que las normas, son instrumentos para la consecución de fines (valores), en un determinado sistema social, piénsese en una pandilla, en un equipo de futbol de nuestro barrio, hasta en un Estado; estos tienen que regirse a través de normas para la consecución de fines; de lo contrario no tendrían funcionamiento.

La justificación ideológica concreta imperante de la Ley y su Reglamento consideramos que es, que a través del Derecho a la consulta (con o sin acuerdo) se busca la inclusión en los procesos de toma de decisión del estado, es decir la participación de los pueblos indígenas y tribales, esa es la razón por el que se emite dicha ley, tolerando sus costumbres. la idea cen- tral es la inclusión, esta idea da cabida a muchos personajes con un discurso de izquierda tradicional (dogmático), a la resistencia radical.

Sin embargo el discurso de inclusión (a través del diálogo intercultural) no es más que un discurso que legitima las decisiones del Estado, solo respecto a las políticas públicas con contenido económico, específicamente del "crecimiento económico". Nos llama la atención lo que sucede en la realidad, aunque la norma establece que se "incluye" a los indígenas en el "proceso" (ojo Proceso) de toma de decisión, lo cierto es que la inclusión se da cuando se ha adoptado una decisión legislativa o administrativa, es decir ya hay una agenda preestablecida.

Para Roger Merino (2015), el verdadero sentido de esta Ley de Consulta es que el Estado establece políticas de desarrollo y mediante el proceso de consulta se tratará de convencer a los "otros" que esas políticas son las más adecuadas para que de esta manera puedan ser "incluidos" en ellas. Para decirlo más abiertamente: el proceso de consulta sirve para legitimar una agenda ya establecida, y si no les gusta, mala suerte.

El problema es que preexiste una visión ideológica de Desarrollo (neoliberal), al cual consideran como perfecto; y por lo tanto las demás ideas quedan descartadas. Además, no se tiene en cuenta la historia y reciente en la que el disenso y no el consenso o acuerdo al que hace tanto hincapié la Ley ha sido el factor homogéneo de la relación Estado - indígenas. 
Por su parte, Alberto Chirif (2015) menciona que La ley de consulta contiene una serie de medidas que modifican el espíritu del derecho de consulta expresado en el Convenio 169. Mientras las organizaciones plantearon que la consulta sea previa a la celebración de contratos que otorguen derechos de exploración o explotación de recursos naturales y que también los estudios de impacto ambiental fuesen sometidos a procesos de consulta antes de su aprobación; el Estado sostuvo e impuso en la ley que la consulta fuese antes del inicio de las actividades de una empresa pero después de la firma de los contratos de exploración y explotación. Con esto, evidentemente la convirtió en una "ley de consulta posterior".

Esta afirmación es alarmante, pero Chirif no menciona ningún artículo en específico; sin embargo creemos que el articulo el cual respalda dicha afirmación es el artículo 3 del Reglamento inciso i) el cual define a las "Medidas administrativas", que deben ser consultadas como, Normas reglamentarias de alcance general; así como el acto administrativo que faculte el inicio de la actividad o proyecto, o el que autorice a la Administración la suscripción de contratos con el mismo fin.

Se debe advertir que la norma tiene como referente al Acto administrativo que: 1) faculte el inicio de la actividad; 2) faculte el inicio del proyecto. 3) autorice a la administración la suscripción de contratos para el inicio de la actividad, 4) autorice a la administración la suscripción de contratos para el inicio del proyecto. En el caso de una concesión minera, el acto administrativo que autorice a la administración la suscripción de contratos para el inicio de la actividad, supone que ya hubo previamente una actividad de exploración (contrato de exploración y explotación); por lo que se concluye la consulta no es más que "un saludo a la bandera".

Asimismo otro problema que se ha advertido es que La Ley supone que los pueblos indígenas y el Estado están en una situación de igualdad, por lo que pueden llegar a un acuerdo a través de la negociación "libre e informada" -idea básica del Análisis Económico del Derecho (AED), ¿será coincidencia? ¿Homo economicus?esta afirmación se desprende de La Ley al señalar como principios la Ausencia de coacción o condicionamiento y la Información oportuna (art. 4 inc.6 y 7); no es plausible que así se llegue a un consenso o acuerdo propiamente, más aun cuando la desigualdad se percibe por doquier y los pueblos indígenas lo viven en carne propia.

Tomando en consideración, alguno de sus postulados, que la corriente neoliberal predica la Liberalización del comercio y la Liberalización de la inversión extranjera, por lo que poco o nada debería interesarle al Estado respecto a la inversión, y solo debería dedicarse a la protección de la propiedad privada (postura de Hernando De Soto) y que debería flexibilizar las normas que impidan la entrada en los mercados; definitivamente La ley es 
utilizada como un mecanismo de legitimación de la política económica que ayudará muy poco en la solución de conflictos sociales; consideramos que La Ley debería ser un mecanismo para construir relaciones democráticas (en todas sus dimensiones) entre el gobierno y los pueblos indígenas.

¡Peor es nada! Debemos aceptarlo respecto al Convenio 169, pero una política integral, ideológicamente coherente, sustentada en las Ciencias Sociales, de verdadera protección de los pueblos indígenas no puede solamente sustentarse en la consulta (formalmente); además, en beneficio de todos los ciudadanos de nuestro país, debe repensarse la actual ideología política económica que genera dependencia en la actividad extractivista, pobreza, inestabilidad económica a largo plazo y contaminación ambiental. Esto se demuestra con los siguientes datos, que encontramos por intermedio del artículo titulado "La mermelada de De Soto" de Alberto Chirif (2015), el Instituto Nacional de Estadísticas e Informática señala: los distritos más pobres de la región Puno son aquellos donde se explota algún mineral. Por ejemplo, en Pichacani-Laraqueri (Puno) el $82.7 \%$ de sus pobladores son pobres y $37.8 \%$ están en pobreza extrema; mientras que en San Antonio de Esquilache (Puno) la pobreza es de $87.2 \%$ y la pobreza extrema $49.9 \%$. Y también la información sobre salud y educación en la Encuesta demográfica y de salud familiar. Nacional y departamental del Instituto Nacional de Estadística e Informática (Lima, 2003). Allí podrán ver que los departamentos con actividad extractiva más antigua (Puno, Junín, Pasco, Cajamarca, Loreto y otro) presentan los peores índices de "Prevalencia de anemia en niñas y niños de 6 a 59 meses de edad (2012)", "Prevalencia de anemia en mujeres de 15 a 49 años de edad (2012), y "Desnutrición entre menores de cinco años de edad" (2012).

\section{4. ¿APARIENCIA O REALIDAD? VALORANDO LA DECISIÓN QUE SE DA EN EL TRÁMITE DE LA CONCESIÓN}

Si a un pueblo, donde no concurran positivamente los seis factores que intervienen en el proceso de consulta, se les efectúa la consulta respectiva y responden afirmativamente, de seguro la pregunta intuitiva que nos surgirá será ¿̨habrá sido ese proceso de consulta uno real -esto es, una decisión libre e informada de los puebloso una mera apariencia? Y debemos responderla situándonos más en el plano del deber ser, que en el del ser. Puesto que allí se verá con mayor nitidez el problema de la consulta previa.

Un ejemplo de lo que tratamos de explicar, sería lo que sucede, en el ámbito jurídico (sobre todo en el Derecho civil), con la patología contractual denominada "lesión", que se configura cuando una persona estando en una necesidad apremiante y urgente de un bien " $A$ " vende parte de sus bienes " $B$ "-que no lo hubiese hecho de no presentarse dicha necesidad y urgencia-, para obtener dicho bien "A" que desea. 
Habiendo llegado hasta aquí, con todo lo expuesto y con este último ejemplo. Si algo realmente queremos, es que el lector haya comprendido que este problema. Que no es otro, que el proceso de consulta previa es una mera apariencia. No es una elucubración errónea nuestra, sino un problema que -desde un enfoque deber serreclama una solución. Que llegado su momento, deberá apoyarse en la ciencia y tecnología social; así como en una determinada ideología, que esté dispuesta a fortalecer todos los factores que intervienen en el proceso de consulta previa.

\section{CONCLUSIONES}

A continuación arribamos a las siguientes conclusiones:

1. La decisión que se da en el proceso de consulta: es una mera apariencia. Puesto que, la concurrencia negativa de los factores que intervienen en el proceso de consulta -que fueron desarrollados en este trabajo-, alteran no solo la libertad del pueblo consultado.

2. La decisión que adopten los pueblos no es vinculante; por tanto, no hay derecho de veto.

3. Según la Ley que regula la consulta previa, la finalidad de la consulta es arribar a un acuerdo, al que debe llegarse, a través de una negociación libre e informada; sin embargo, creemos que no es tan cierto, puesto que, son necesarios, pero no son suficientes.
Por lo que, los factores que se deben tener en cuenta y se deben fortalecer son: el económico, educativo, el factor seguridad, el factor alimenticio, político y ambiental.

4. La ideología imperante, en la que se justifica la consulta previa, es el neoliberalismo. El cual refuerza la liberalización del comercio y la liberalización de la inversión extranjera. Esta ideología no se preocupa por los factores distintos al económico (predica el economicismo).

5. La Ley de Consulta previa, en ciertos aspectos es un obstáculo para las políticas públicas, guiados por la ideología imperante, que se vienen implementando; sin embargo, al mismo tiempo es también un instrumento para los fines de dicha ideología.

\section{BIBLIOGRAFÍA}

1. D.S. No 001-2011-MC. (2 de Abril de 2011). Reglamento de la Ley a la consulta previa a los pueblos indigenas $u$ originarios reconocido en el convenio 169 de la Organizacion Internacional del Trabajo (OIT). Lima.

2. Bunge, M. (1985). Seudociencia e ideología. Madrid: Alianza Editorial.

3. Bunge, M. (1999). Buscar la filosofía en las ciencias sociales. España: Siglo XXI editores.

4. Bunge, M. (2009). Filosofia Po- 
lítica: Solidaridad, Cooperación y Democracia Integral (Primera ed.). (R. Gonzales del Solar, Trad.) Barcelona: Gedisa.

5. Bunge, M. (2012). Tratado de Filosofía. Ontología II: un mundo de sistemas (Primera ed., Vol. IV). (R. González del Solar, Trad.) Barcelona: Gedisa.

6. Chirif, A. (22 de Julio de 2015). Cecopros. Obtenido de http://cecopros.org/principal//content/ view/1158/308/.

7. Chirif, A. (22 de julio de 2015). La mula. Obtenido de https://redaccion.lamula.pe/2015/04/23/ los-20-anos-del-convenio169-de-la-oit-en-el-peru-hay-algo-que-celebrar/albertoniquen/

8. Damonte, G., \& Ramírez, T. (Enero de 2012). Ley de consulta: aváncez, problema y posibilidad. Perú debate(2), 18-23.

9. Flores, C. (octubre de 2011). El Derecho a la Consulta Previa y el Convenio 169 OIT. Recuperado el 2 de mayo de 2016, de El Derecho a la Consulta Previa y el Convenio 169 OIT: http://docplayer.es/12493736-El-derechoa-la-consulta-previa-y-el-convenio-169-oit.html
10. Gallardo, J. M. (10 de Noviembre de 2014). El diario de Chemazdamundi. Obtenido de https://chemazdamundi.wordpress.com/2014/11/10/el-neoliberalismo-contrastado-v-ensayo-sobre-el-neoliberalismo/

11. Harvey, D. (2007). Breve historia del Neoliberalismo. Madrid: Akal.

12. Ley $\mathrm{N}^{\circ}$ 29785. (30 de Agosto de 2011). Ley del derecho a la consulta previa a los pueblos indigenas $u$ originarios, reconocido en el convenio 169 de la organizacion internacional del trabajo (OIT). Lima.

13. Merino, L. B., \& Lanegra, I. (2013). Consulta previa a los pueblos indígenas. El desafio del diálogo intercultural en el Perú. (Primera edición ed.). Perú: CENTRUM Católica-Centro de Negocios de la Pontificia Universidad.

14. Merino, R. (20 de Julio de 2015). Blog: Comparación Jurídica y Análisis Crítico. Obtenido de Minorias o Naciones.: http://comparacioncritica.blogspot.com/

15. Pinto, H. (2013). Conflicto minero en Santa Ana - Puno. Investigaciones Sociales, 17(31), 207-220. 